\title{
Avian influenza - The next pandemic?
}

\author{
John M Conly $M D^{1}$, B Lynn Johnston $M D^{2}$
}

Arepr vian influenza (AI) strains affecting humans were first reported in Hong Kong during the 1997 influenza season. These strains were shown to be derived from avian sources without genetic reassortment between avian and human influenza viruses (1-3). The first AI A strain (H5N1) affected 18 humans and was associated with six deaths during the 1997 to 1998 influenza season. A case-control study (4) identified recent exposure to live poultry as an important risk factor for infection with H5N1. Furthermore, cohort studies $(5,6)$ suggested that human-to-human transmission of the H5N1 virus was limited. Three additional cases of confirmed H5N1 influenza occurred in Hong Kong in February 2003, causing two deaths (7). From late 2003 to February 2004, outbreaks of $\mathrm{H} 5 \mathrm{~N} 1$ infection in poultry were detected in a number of Asian countries, notably Vietnam, Thailand, Cambodia, China, Indonesia, Japan, Laos and the Republic of Korea (8). These poultry outbreaks have been unpredictable and unprecedented in their scope, with wide geographical spread and severe economic consequences for the affected countries. More than one-half of the aforementioned countries experienced highly pathogenic $\mathrm{AI}$ in fowl for the first time in their respective histories.

The poultry outbreak in 2004 also affected humans, resulting in the largest recorded human outbreak of $\mathrm{H} 5 \mathrm{~N} 1$ influenza to date (34 officially reported cases, 23 of which were fatal) (9). Although no human cases had been reported since the winter of 2003/2004, the World Health Organization reported three fatal human cases of laboratory-confirmed AI in Vietnam in August 2004, with H5N1 identified as the etiological agent (10). In addition, the AI A H9N2 virus was isolated for the first time in two young, hospitalized children in Hong Kong, in March 1999 (11,12). The children were unrelated, hospitalized at different facilities, did not have any known contact with or link to each other and had not travelled outside Hong Kong. Both children had uncomplicated, febrile, upper respiratory tract illness. Evidence for five additional human infections attributable to the H9N2 influenza virus was reported from the Guangdong province of China in 1998 (13). The presence of the H9N2 antibody from persons in mainland China and poultry workers in Hong Kong $(13,14)$ suggests that additional unrecognized human $\mathrm{H} 9 \mathrm{~N} 2$ infections have occurred. Another strain, AI H7N7, has been isolated in humans on three separate occasions since 1980 . The majority of these patients developed conjunctivitis after contact with harbour seals, ducks or chickens. In addition, an outbreak of AI H7N7 in the Netherlands was associated with the death of a veterinarian in April 2003. This outbreak was also associated with mild illness in 83 humans, the majority of whom had conjunctivitis (although seven exhibited respiratory symptoms). Mild cases of infection due to an H9N2 strain occurred in children in Hong Kong in 1999 (two cases) and in mid-December 2003 (one case). Given the repeated occurrences of human AI in Asia and Europe, it was considered timely to review the current taxonomy and epidemiology of AI, and the risks these strains pose to the human population.

Influenza $\mathrm{A}$ and $\mathrm{B}$ are the two types of influenza viruses responsible for human epidemic disease. Influenza type C viruses cause mild illness in humans and do not appear to cause epidemics or pandemics. Influenza A viruses are found in many different animal species, including humans, ducks, chickens, pigs, whales, horses and seals, whereas influenza B viruses circulate widely only among humans. Influenza A viruses are divided into subtypes based on two antigens (hemagglutinin $[\mathrm{H}]$ and neuraminidase $[\mathrm{N}]$ ) on the surface of the virus, whereas influenza B and $\mathrm{C}$ viruses are not classified according to subtype. There are 15 different hemagglutinin subtypes and nine different neuraminidase subtypes identified to date, all of which have been found among influenza $A$ viruses in wild birds. The hemagglutinin acts as a site of attachment of the virus to host cells to initiate infection and also to erythrocytes, from which its name originally was derived (15). Hemagglutinin contains common and strain-specific antigens and demonstrates antigenic variation. The neuraminidase antigen contains subtype-specific antigens, and also demonstrates antigenic variation between subtypes. Neuraminidase is a surface glycoprotein possessing enzymatic activity essential for viral replication in influenza A and influenza B viruses. Neuraminidase allows the release of newly produced virions from infected host cells, prevents the formation of viral aggregates after release from the host cells and prevents viral inactivation by respiratory mucous $(16,17)$. It is thought that this enzyme may also promote viral penetration into respiratory epithelial cells, and may contribute to the pathogenicity of the virus by promoting the production of pro-inflammatory cytokines such as interleukin-1 and tumour necrosis factor from macrophages (18-20).

\footnotetext{
${ }^{1}$ Departments of Pathology and Laboratory Medicine, Medicine, and Microbiology and Infectious Diseases, Centre for Antimicrobial Resistance, University of Calgary, Calgary, Alberta; ${ }^{2}$ Queen Elizabeth II Health Sciences Centre and Dalhousie University, Halifax, Nova Scotia Correspondence: Dr JM Conly, Foothills Medical Centre, 1403-29 Street South, Calgary, Alberta T2N 2T9. Telephone 403-944-8222, fax 403-944-1095, e-mail jconly@ucalgary.ca
} 
The influenza A virus affecting humans has traditionally involved three subtypes based on one of three hemagglutinin antigens ( $\mathrm{H} 1, \mathrm{H} 2$ and $\mathrm{H} 3)$ and two additional subtypes based on one of two neuraminidase antigens (N1 or N2). However, in the past decade, strains of influenza virus containing other $\mathrm{H}$ and $\mathrm{N}$ antigenic combinations have affected humans (as noted previously), with $\mathrm{H} 5 \mathrm{~N} 1$ strains having the highest reported rates of fatality. Wild birds are the primary natural reservoir for all subtypes of influenza $A$ viruses and are thought to be the source of influenza A viruses in other animals. Most influenza viruses cause asymptomatic or mild infection in wild birds; however, the range of symptoms in birds varies greatly depending on the strain of virus. Domestic poultry, such as turkeys and chickens, can become seriously ill and eventually die from AI. In addition, some avian viruses can cause serious disease and death in wild birds. AI, or 'fowl plague', has been recognized since the late 1800s and in the latter part of the last century, it was recognized as a member of the influenza virus family. At one time, it was thought that all virulent fowl influenza was caused by influenza A viruses containing the $\mathrm{H} 7$ antigen; however, it was recognized that $\mathrm{H} 5$ and other $\mathrm{H}$-containing subtypes had been associated with highly virulent influenza virus infections in fowl, and that $\mathrm{H} 7$ did not always cause virulent disease. AI viruses are classified based on virulence (21). AI A viruses (eg, some strains of H5 and H7 viruses) that cause widespread disease and death among fowl populations are considered highly pathogenic AI (HPAI). HPAI viruses can kill $90 \%$ to $100 \%$ of infected chickens, whereas low pathogenic AI viruses cause less severe or no illness if they infect chickens. Although HPAI is often associated with high mortality in poultry, it is not certain how the distinction between low and high pathogenicity is related to the risk of disease in humans. Low pathogenic AI viruses may also evolve into HPAI viruses. Birds that survive influenza infection continue to shed virus via the fecal-oral route for at least 10 days, thus allowing further viral transmission by migratory birds or in crowded poultry markets.

As noted, the H5N1 AI strains first reported from Hong Kong during the 1997 to 1998 influenza season were shown to be derived from avian sources without genetic reassortment between avian and human influenza viruses (1). The H5N1 isolates from patients appeared identical to the HPAI H5N1 strain found in chickens during an outbreak in Hong Kong earlier that year. Evidence suggested that close contact with viruscontaminated birds in live poultry markets was the source of the H5N1 outbreak (21). AI viruses do not replicate efficiently in humans and direct person-to-person transmission between humans has not been demonstrated (22).

Although current evidence suggests that these strains of the AI virus were transmitted bird-to-human and are not efficiently transmitted from person-to-person, the implications of these avian outbreaks for human health are highly significant. Outbreaks of the H5N1 strain in the human population have now caused limited but severe disease with high fatality on four separate occasions since 1997. The human outbreak of 2004 was the largest ever recorded, resulting in 34 officially reported cases ( 23 of which were fatal). These human cases of H5N1 were not confined to any demographic group. The Hong Kong outbreak of 1997 saw illness develop in patients ranging from one to 60 years of age. Similar to traditional influenza in humans, older adults had more severe disease and children had less severe disease. However, in Thailand during early 2004, four of the first five $\mathrm{H} 5 \mathrm{~N} 1$ patients were children less than seven years of age, all of whom died. All of the patients were previously healthy but had links to contact with poultry. As such, the full extent of the epidemiology of human AI cases has not yet been fully elucidated. Additional concerns have surfaced, including the lack of a vaccine and issues surrounding delayed treatment of severe illness due to the inability to execute rapid laboratory identification of the virus. Perhaps the greatest concern is the possible emergence of a new virus subtype with pandemic potential. Pandemics recur unpredictably and are associated with high morbidity and mortality, and great social and economic disruption.

Considering the widespread nature of the current H5N1 outbreak in Asia and the ability of influenza viruses to jump the species barrier, it is inevitable that the $\mathrm{H} 5 \mathrm{~N} 1$ virus will be detected in pigs. The cocirculation of avian, human and pig viruses in pigs is of significant concern because of the potential for a genetic exchange, or 'reassortment', of material between these viruses (23). The chances for genetic reassortment depend on the duration of $\mathrm{H} 5 \mathrm{~N} 1$ circulation in pigs and the simultaneous presence of human and pig influenza A viruses (such as H3N2 or H1N1). Such an occurrence has the potential to produce a new pandemic influenza strain. Pigs can be infected with both avian and human influenza A viruses - for instance, human influenza H3N2 viruses have been detected in pigs in Asia, Europe and Africa. Some of these human and AI viruses might become adapted to pigs and then begin circulating in pig populations. The dense cohabiting populations of humans, pigs and wild and domestic fowl that exists in China permits favourable conditions for genetic reassortment of viruses from different species, which could potentially lead to a pandemic influenza strain.

Adding further to this concern, a recent report from China's Harbin Veterinary Research Institute announced that pigs from farms in parts of China had been infected with the H5N1 strain of AI (23). China's Ministry of Agriculture has since confirmed these findings. What is unclear from the few studies that have been conducted is whether the $\mathrm{H} 5 \mathrm{~N} 1$ virus is already established in pig populations in China (23).

Assessing the consequences of this information for public health is difficult because these findings remain preliminary and are not necessarily indicative of widespread infection among pigs. Providing a detailed risk assessment of the current situation requires an understanding of the main factors influencing the potential for the emergence of a pandemic influenza strain: the prevalence of $\mathrm{H} 5 \mathrm{~N} 1$ and human $\mathrm{H} 3 \mathrm{~N} 2$ virus in pigs in Asia, and the likelihood of a reassorted virus and its possible pathogenicity; however, all of these factors remain unknown entities. In addition, the role of pigs in genetic reassortment is not fully understood.

To better understand the implications of the findings in China, the World Health Organization is encouraging the conduct of additional studies on $\mathrm{H} 5 \mathrm{~N} 1$ and other influenza A viruses in pigs in China and other countries experiencing H5N1 AI outbreaks. In addition, laboratory experiments are required to shed some light on the probability of virus reassortment, the possible pathogenicity of a reassorted virus and the chance that pigs will act as a pathway for the emergence of a potential pandemic strain. These results will help national and international public health authorities assess the role pigs and humans play in the potential emergence of a new influenza 
pandemic $\mathrm{H} 5 \mathrm{~N} 1$ virus and structure the necessary public health interventions.

Some influenza experts believe that strategies may be effective in reducing the opportunity for human exposure to the AI virus. These strategies, which include prompt culling of infected flocks, vaccination and protection of persons at high risk of exposure to infected poultry and optimizing the use of existing vaccines effective against currently circulating human influenza strains, could reduce the likelihood of coinfection of humans with avian and influenza strains, thus reducing the risk of reassortment (23). Whether these strategies can reduce the likelihood of an emergent pandemic strain, and potentially avert an influenza pandemic, remains to be seen.

\section{REFERENCES}

1. Centers for Disease Control and Prevention. Update: Isolation of avian influenza A (H5N1) viruses from humans - Hong Kong, 1997 to 1998. MMWR Morb Mortal Wkly Rep 1998;46:1245-7.

2. Claas ECJ, Osterhaus ADME, van Beek R, et al. Human influenza A H5N1 virus related to a highly pathogenic avian influenza virus. Lancet 1998;351:472-7.

3. Lee SY, Mak KH, Saw TA. The avian flu (H5N1): One year on. Department of Health, Hong Kong Special Administrative Region of China. Public Health and Epidemiology Bulletin 1999;8:1-7.

4. Mounts AW, Kwong H, Izurieta HS, et al. Case-control study of risk factors for avian influenza A (H5N1) disease, Hong Kong, 1997. J Infect Dis 1999;180:505-8.

5. Buxton BC, Katz JM, Seto WH, et al. Risk of influenza A (H5N1) infection among health care workers exposed to patients with influenza A (H5N1), Hong Kong. J Infect Dis 2000;181:344-8.

6. Katz JM, Lim W, Bridges CB, et al. Antibody response in individuals infected with avian influenza A (H5N1) viruses and detection of anti-H5 antibody among household and social contacts. J Infect Dis 1999;180:1763-70.

7. Nicholson KG, Wood JM, Zambon M. Influenza. Lancet 2003;362:1733-45.
8. World Health Organization. Avian influenza A (H5N1) Update 32: Situation (human) in Thailand.

$<$ http://www.who.int/csr/don/2004_03_09/en> (Version current at September 14, 2004)

9. Assessment of risk to human health associated with outbreaks of highly pathogenic H5N1 avian influenza in poultry - Situation as at 14 May 2004. Wkly Epidemiol Rec 2004;79:203-4.

10. World Health Organization. Avian influenza - Situation in Vietnam as of 18 August 2004. Wkly Epidemiol Rec 2004;79:309-12.

11. World Health Organization. Influenza. Wkly Epidemiol Rec 1999;74:111.

12. Peiris M, Yuen KY, Leung CW, et al. Human infection with influenza H9N2. Lancet 1999;354:916-7.

13. Guo YJ, Li J, Cheng X, et al. Discovery of man infected by avian influenza virus. Chin J Exp Clin Virol 1999;13:105-8.

14. Shortridge KF. Pandemic influenza: A zoonosis? Semin Respir Infect 1992;7:11-25.

15. Treanor J. Influenza virus. In: Mandell GL, Douglas RG, Bennett JE, eds. Principles and Practices of Infectious Diseases, 5th edn. New York: Churchill-Livingston, 2000:1823-49.

16. Liu C, Air GM. Selection and characterization of a neuraminidaseminus mutant of influenza virus and its rescue by cloned neuraminidase genes. Virology 1993;194:403-7.

17. Buck P, Herman S, Scott C, et al. Respiratory virus surveillance. FluWatch Project, 1997-1998. Can Commun Dis Rep 1998;24:49-51.

18. Colman PM. Neuraminidase: Enzyme and antigen. In: Krug RM, ed. The Influenza Viruses. New York: Plenum Press, 1989:175-218.

19. Colman PM. Influenza virus neuraminidase: Structure, antibodies and inhibitors. Protein Sci 1994;3:1687-96.

20. Schultz-Cherry S, Hinshaw VS. Influenza virus neuraminidase activities latent transforming growth factor beta. J Virol 1996;70:8624-9.

21. Horimoto T, Kawaoka, Y. Pandemic threat posed by avian influenza A viruses. Clin Microbiology Rev 2001;14:129-49.

22. Capua I, Alexander DJ. Avian influenza and human health. Acta Trop 2002;83:1-6.

23. World Health Organization. Avian influenza - Current evaluation of risks to humans from H5N1 following recent reports. Wkly Epidemiol Rec 2004;79:265-72. 


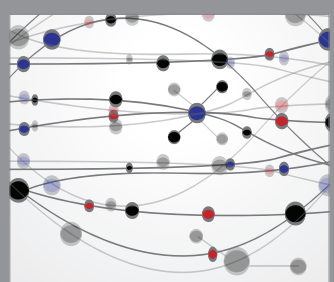

The Scientific World Journal
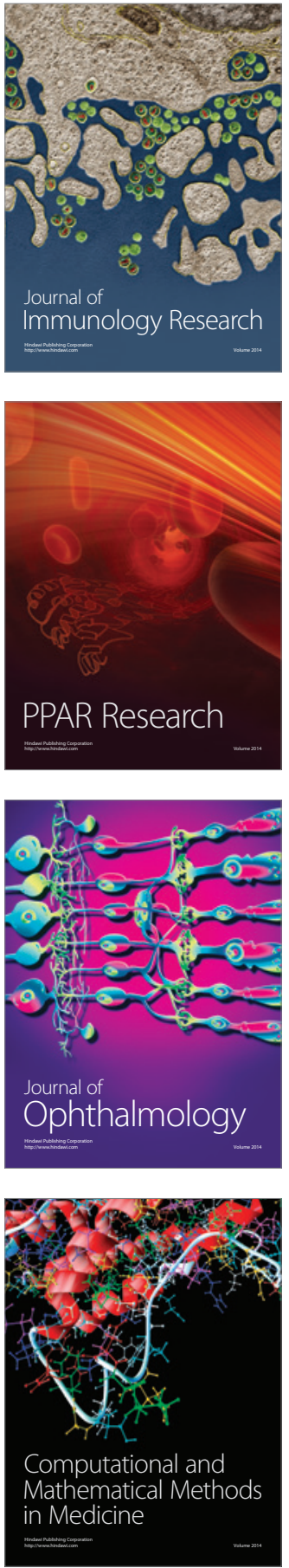

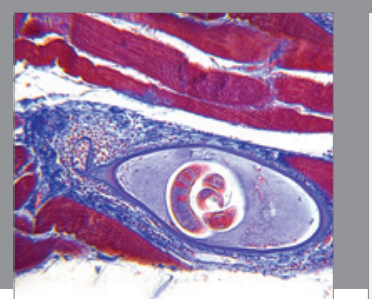

Gastroenterology Research and Practice

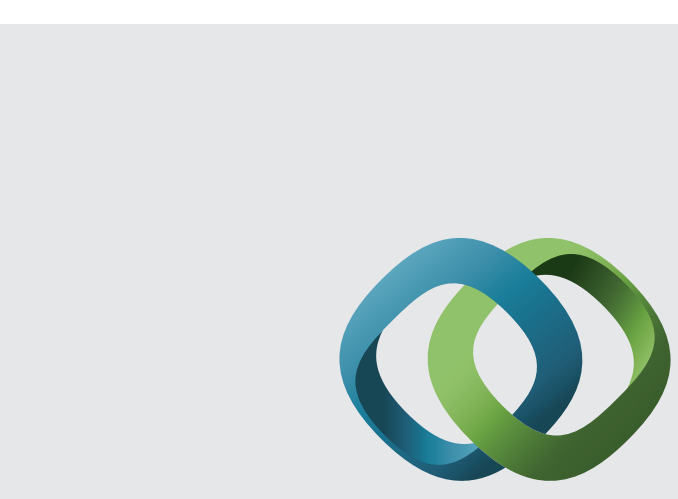

\section{Hindawi}

Submit your manuscripts at

http://www.hindawi.com
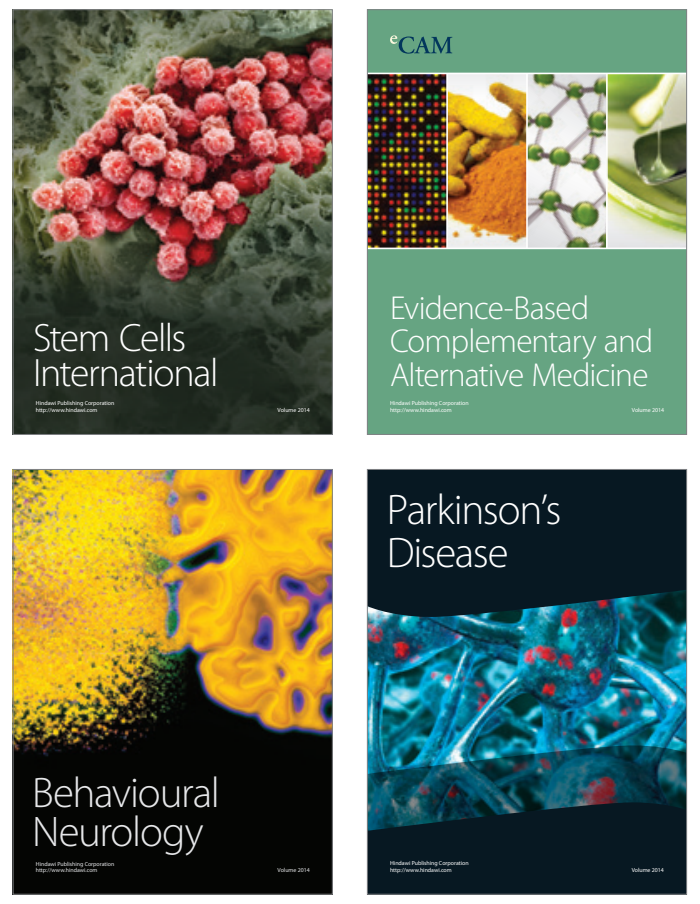
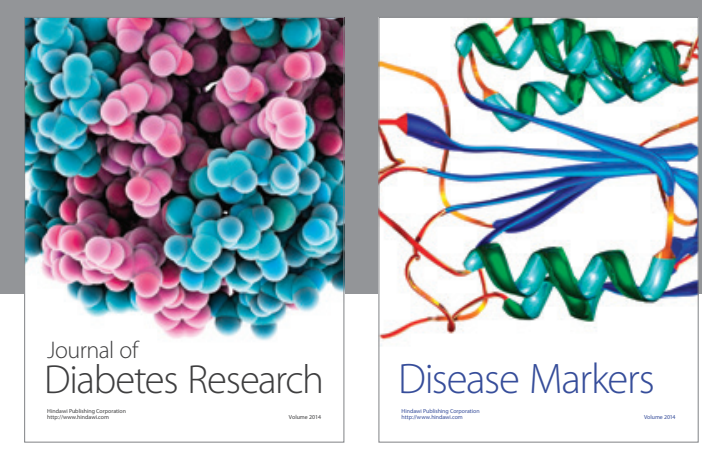

Disease Markers
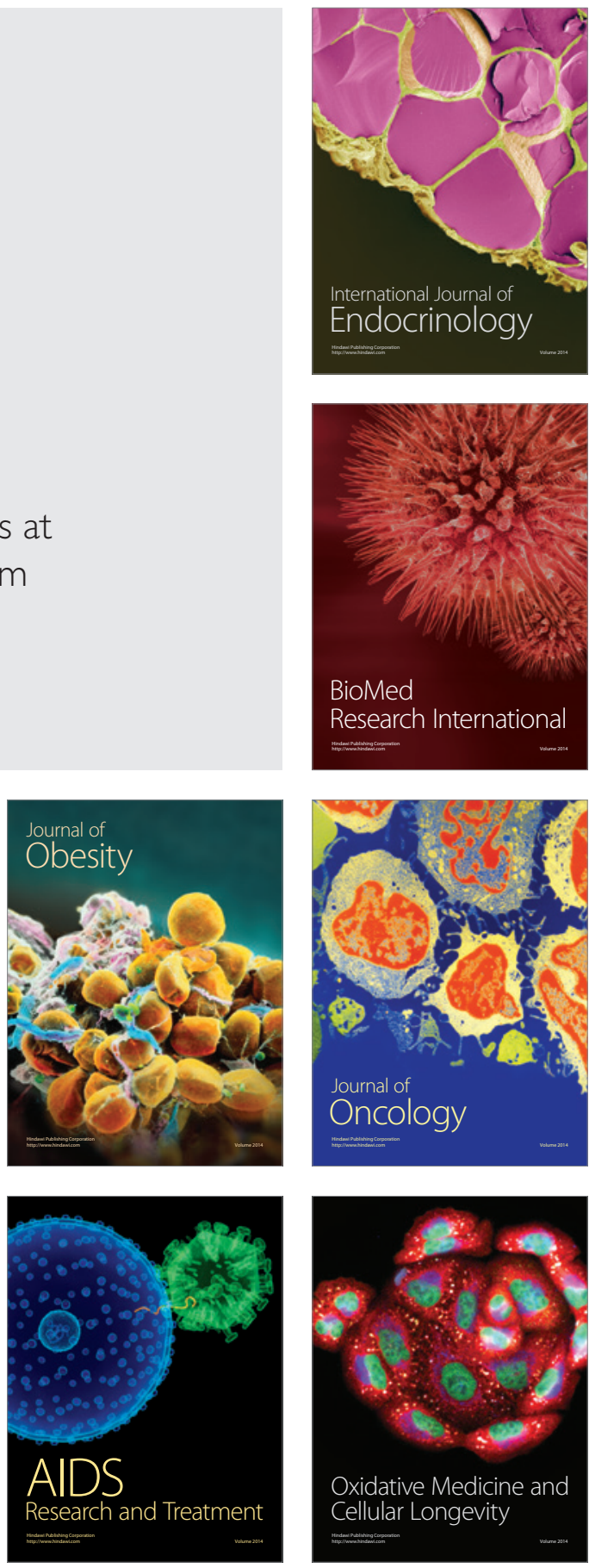